\title{
Desenvolvimento e validação de um jogo de tabuleiro para crianças com câncer
}

Development and validation of a board game for children with cancer Desarrollo y validación de un juego de mesa para niños con cáncer

Daniela Doulavince Amador ${ }^{1}$ (i) https://orcid.org/0000-0003-0641-1743 Myriam Aparecida Mandetta² iD https://orcid.org/0000-0003-4399-2479

Como citar: Amador DD, Mandetta MA. Desenvolvimento e validação de um jogo de tabuleiro para crianças com câncer. Acta Paul Enferm. 2022;35:eAPE00121.

DOI

http://dx.doi.org/10.37689/acta-ape/2022A000121

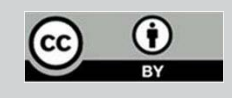

Descritores

Criança; Neoplasias; Comunicação; Jogos e brinquedos

Keywords

Child; Neoplasms; Communication; Play and plaything

Descriptores

Niño: Neoplasias; Comunicación; Juego e implementos de juego

$$
\begin{array}{r}
\text { Submetido } \\
24 \text { de Janeiro de } 2020 \\
\text { Aceito }
\end{array}
$$

26 de Maio de 2021

\section{Autor correspondente} Daniela Doulavince Amado E-mail: dani_doulavince@yahoo.com.br

Editor Associado (Avaliação pelos pares): Edvane Birelo Lopes De Domenico (https://orcid.org/0000-0001-7455-1727) Escola Paulista de Enfermagem, Universidade Federal de São Paulo, São Paulo, SP, Brasi

\section{Resumo}

Objetivo: Elaborar e validar um jogo de tabuleiro para comunicação efetiva entre profissionais da saúde e crianças com câncer.

Métodos: Pesquisa metodológica que utilizou a teoria socioconstrutivista de Vigostky como referencial teórico e o Child-Centered Game Development (CCGD), como referencial metodológico. 0 estudo foi realizado entre os meses de fevereiro/2016 e julho/2017 e seguiu as seguintes etapas: análise, conceito, design, implementação e avaliação. Nas fases que a criança participou foram incluídas aquelas com idade entre 8 e 12 anos, diagnosticadas com câncer. Durante a construção do jogo, os dados coletados foram analisados de acordo com o objetivo de cada etapa. A pesquisa foi aprovada pelo Comitê de Ética em Pesquisa de um Instituto de Ensino Superior (Parecer: 1.387.962/2014).

Resultados: 0 estudo de fase de análise mostrou que a criança deseja ser informada numa linguagem adequada ao seu nível de compreensão. Na fase de conceito foram definidos os elementos de desenvolvimento do jogo. Na fase de design foi proposto um protótipo do tabuleiro do jogo que, em seguida passou pela fase de implementação para que a criança pudesse fazer uma avaliação mais fidedigna do design do jogo de tabuleiro. Na fase de avaliação, o conteúdo das cartas foi validado pelos profissionais com índice geral de 0,95 , os designers em jogos aprovaram a usabilidade e as crianças relataram apreciar o jogo.

Conclusão: 0 jogo de tabuleiro "Skuba! Uma aventura no fundo do mar" foi considerado validado. Dessa maneira, o jogo se constitui uma importante ferramenta no processo comunicacional com essa populaçãoalvo.

\section{Abstract}

Objective: To elaborate and validate a board game for effective communication between health professionals and children with cancer.

Methods: This methodological research followed Vigostky's social-constructivist theory as a theoretical framework and the Child-Centered Game Development (CCGD) as a methodological framework. We carried out the study between February/2016 and July/2017 and followed these phases: analysis, concept, design, implementation, and evaluation. In the phases that we had children's participation, we included those who were between 8 to 12 years old and diagnosed with cancer. During the development of the game, we analyzed the collected data according to the objective of each phase. The Research Ethics Committee from a Higher Education Institute approved the research (Protocol number: 1.387.962/2014).

Results: The phase study revealed that the child wishes to be informed in an adequate language to their level of comprehension. In the concept phase, we defined the developing elements of the game. In the design 
phase, we propose a prototype of the board that subsequently was implemented so that the child could carry out a reliable evaluation of the game board design. In the evaluation phase, professionals evaluated the content of the cards with an average index of 0.95 , the game designers approved the usability, and the children reported enjoying the game.

Conclusion: We considered the game board "Skuba! An adventure under the sea" valid. Thus, the game represents an important tool in the communication process with this target audience.

\section{Resumen}

Objetivo: Elaborar y validar un juego de mesa para una comunicación efectiva entre profesionales de la salud y niños con cáncer.

Métodos: Estudio metodológico que utilizó la teoría socioconstructivista de Vigostky como marco referencial teórico y el Child-Centered Game Development (CCGD) como marco referencial metodológico. El estudio fue realizado entre los meses de febrero de 2016 y julio de 2017 y completó las siguientes etapas: análisis, concepto, diseño, implementación y evaluación. En las fases que hubo participación de niños, se incluyeron aquellos entre 8 y 12 años de edad, diagnosticados con cáncer. Durante la elaboración del juego, los datos recopilados fueron analizados de acuerdo con el objetivo de cada etapa. El estudio fue

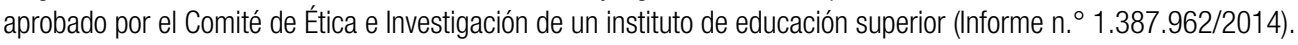

Resultados: El estudio en fase de análisis demostró que los niños desean ser informados con un lenguaje adecuado a su nivel de comprensión. En la fase de concepto, se definieron los elementos de desarrollo del juego. En la fase de diseño, se presentó un prototipo del tablero del juego, que luego pasó a la fase de implementación para que los niños pudieran hacer una evaluación más fidedigna del diseño del juego de mesa. En la fase de evaluación, los profesionales validaron el contenido de las cartas con un índice general de 0,95, los diseñadores de juegos aprobaron la usabilidad y los niños relataron que el juego les gustó.

Conclusión: El juego de mesa "¡Skuba! Una aventura en el fondo del mar" fue considerado validado. De esta manera, el juego constituye una herramienta importante en el proceso comunicativo con el público destinatario.

\section{Introdução}

Crianças e adolescentes com câncer tem expressado, cada vez mais em pesquisas, que desejam ser informadas a respeito da doença e do seu tratamento. ${ }^{(1-5)}$ Sabe-se que a maneira como a criança enfrenta o diagnóstico de câncer é, muitas vezes, influenciada pela representação que constrói acerca da doença e pelas relaçóes interpessoais existentes nesse processo. ${ }^{(2,3)}$

A comunicação entre o profissional, a criança e sua família é um instrumento relevante para transformar e ressignificar o sentido atribuído à doença. Entretanto, na maior parte desse processo, a criança é alocada em segundo plano, ou se depara com uma linguagem complexa e uma dificuldade emocional para lidar com tantas informaçôes. ${ }^{(3,5)}$

Comunicar-se de forma efetiva com a criança é proporcionar um ambiente que permita suas expressóes, questionamentos e curiosidades, implicando, assim, em uma conexão entre os interlocutores que torne o processo mais leve por meio da segurança transmitida nesse vínculo.

A utilização de recursos lúdicos, adequados à fase de seu desenvolvimento, amplia as possibilidades para que a criança possa compreender as adversidades do tratamento e, consequentemente, lidar com elas. ${ }^{(6,7)}$ Nesse sentido, os jogos mantêm uma relação estreita com a construção do conhecimento, exercem influência como um elemento motivador e proporcionam um ambiente agradável, planejado e enriquecido, que possibilita a aprendizagem de várias habilidades. ${ }^{(8)}$

$\mathrm{O}$ uso de jogos e de atividades lúdicas pode ser uma ferramenta útil e bem recebida pelas crianças para se atingir o objetivo de comunicação e compartilhamento de informaçóes numa linguagem clara e acessível. Com o uso do jogo, a criança dirige seu comportamento através do significado que a situação vivenciada proporciona e se engaja em atividades por meio das quais podem assumir novas identidades, explorar mundos e aprender brincando. ${ }^{(8-10)}$

Dessa maneira, os jogos de tabuleiro podem ser considerados como uma via de máo dupla na interação com a criança, por meio dos quais o profissional pode se comunicar de forma mais efetiva, com uma linguagem compreensível, ao mesmo tempo em que se constitui um espaço de divertimento para a criança.

A busca por jogos que remetessem a situaçóes que a criança vai enfrentar durante o diagnóstico e o tratamento do câncer tornou-se uma lacuna a ser preenchida, pois entende-se que é preciso propor meios para ajudar a criança com câncer a lidar melhor com a experiência de doença, como a oferta de materiais em uma linguagem acessível e interativa. 
Assim, o objetivo deste trabalho foi elaborar um jogo de tabuleiro para comunicação efetiva entre profissionais da saúde e crianças com câncer e validar o conteúdo das informaçôes textuais e das imagens com a colaboração das crianças e de juízes de diferentes áreas.

\section{Métodos}

Trata-se de uma pesquisa metodológica que utilizou a teoria socioconstrutivista de Vigostky ${ }^{(11)}$ como referencial teórico e o Child-Centered Game Development (CCGD), ${ }^{(12)}$ como referencial metodológico.

A Teoria Sociointeracionista de Lev Semionóvich Vygotsky tem como ponto central as relaçóes sociais, por meio das quais o indivíduo é moldado através de sua relação e interação com o ambiente. ${ }^{(11)} \mathrm{O}$ referencial considera a interação jogador-jogo em que o jogador pode lançar mão de ações como tomada de decisão, escolha, priorização e raciocínio de estratégias de resolução de problemas, através de ferramentas mediadoras. ${ }^{(13)}$

O referencial Child-Centered Game Development (CCGD) considera que para o desenvolvimento de jogos a criança deve participar de todo o processo de criação e validação, de maneira que suas necessidades, crenças e percepçôes sejam incorporadas. Desse modo, as crianças são encorajadas a assumirem o papel de informantes, usuárias, testadoras e, até mesmo, parceiras no design. O CCGD é estruturado em cinco fases, sendo elas: análise, conceito, design, implementação e avaliação. ${ }^{(12)} \mathrm{A}$ construção do jogo ocorreu entre os meses de fevereiro/2016 e julho/2017 e perpassou pelas cinco fases como descrito a seguir.

\section{Fase 1 - Análise}

Nesta fase utilizou-se os resultados de um estudo descritivo de abordagem qualitativa realizado pelas autoras para identificar as necessidades de informação de crianças com câncer em tratamento quimioterápico. ${ }^{(1)} \mathrm{O}$ estudo foi realizado em um hospital referência no tratamento do câncer infantil. Participaram nove crianças com idades de oito a 12 anos, com diagnóstico confirmado de câncer. A coleta de dados foi feita por meio de entrevista semiestruturada e, para análise dos dados, foi utilizada a Análise Qualitativa de Conteúdo. ${ }^{(14)}$

\section{Fase 2 - Conceito}

Realizaram-se reunióes de discussão da equipe composta pelas pesquisadoras e um game designer, a fim de definir o objetivo do jogo e os elementos para sua composição, incluindo-se o público-alvo, o cenário, os personagens, a narrativa, a dinâmica e o conteúdo das cartas do jogo. Alguns conceitos iniciais foram criados por meio de brainstorm das ideias individuais e coletivas da equipe e da inclusão de conteúdos relacionados à comunicação com a criança e suas necessidades de informação, baseado nos dados do estudo de análise. ${ }^{(1)}$

\section{Fase 3 - Design}

Procedeu-se a criação de um protótipo de baixa fidelidade do jogo com base nas discussóes realizadas na fase de conceito. Uma ilustradora realizou o desenvolvimento da arte por meio de técnicas de editoração e desenho de imagens digitais; e um game designer alocou os elementos no tabuleiro utilizando técnicas computacionais de desenvolvimento de jogos.

\section{Fase 4 - Implementação}

Realizou-se a projeção do protótipo na plataforma alvo escolhida, no caso deste estudo, o tabuleiro, utilizando-se o programa Adobe Illustrator (Ai) e impressa uma versão-teste para aplicação. $\mathrm{O}$ jogo implementado no tabuleiro passou por uma validação semântica com o público-alvo, isto é, crianças com câncer. Depois de jogar uma partida completa do jogo, as crianças narravam sua compreensão acerca das cartas, das ilustraçóes e da dinâmica do jogo.

\section{Fase 5 - Avaliação}

Foram realizados três estudos: avaliação da jogabilidade, validação de conteúdo e avaliação da usabilidade. Para avaliação da jogabilidade pelo público-alvo, conduziu-se um estudo do tipo exploratório e descritivo, de natureza qualitativa para explorar 
as experiências da criança durante sua interação com o jogo. Foram adotados os seguintes critérios de inclusão: criança com idade entre 8 e 12 anos, diagnosticadas com câncer há, pelo menos, um mês e em tratamento quimioterápico ambulatorial. Foram excluídas as crianças internadas para tratamento clínico/cirúrgico e/ou diagnosticadas como fora de possibilidades de cura. Os dados foram coletados em um hospital pediátrico que atende crianças com câncer na cidade de São Paulo. A análise de dados das etapas qualitativas foi conduzida a partir da Análise Qualitativa de Conteúdo. ${ }^{(14)}$

Para a validação do conteúdo do jogo de tabuleiro, a versão 1 foi submetida a um comitê de especialistas na área temática, cujo caminho metodológico foi adaptado a partir dos critérios estabelecidos por Pasquali. ${ }^{(15)} \mathrm{O}$ jogo foi avaliado em relação ao objetivo; a estrutura e apresentação; e a relevância. Utilizou-se a técnica Delphi para obtenção do consenso entre os especialistas, estabelecendo-se o nível de $80 \%$. Para determinar a concordância entre eles, considerou-se o Índice de Validade de Conteúdo (IVC) entre os itens de 0,8, apresentado como um valor de excelência. ${ }^{(16)}$ Participaram dessa etapa profissionais da área da saúde e/ou da educação que atendiam os seguintes critérios de inclusão: ter conhecimento sobre o cuidado à criança com câncer e/ou acerca de tecnologias educativas; ter título de mestre e/ou doutor.

A avaliação da usabilidade foi realizada por um comitê de especialistas em design de jogos, aplicando-se as heurísticas de Nielsen adaptadas para jogos de tabuleiros não virtuais. ${ }^{(17)}$ Os autores propóem dez princípios de design chamados de heurísticas: (1) status do sistema, (2) compatibilidade do sistema e o mundo real, (3) controle e liberdade para o usuário, (4) consistência e padróes, (5) prevenção de erros, (6) reconhecimento em lugar de lembrança, (7) flexibilidade e eficiência de uso, (8) estética e design minimalista, (9) auxílio aos usuários a reconhecer, diagnosticar e recuperar erros e (10) ajuda e documentação. Para cada heurística avaliada, a severidade do problema encontrado deveria ser classificada com base em uma escala de 0 a 4 , sendo $0=$ sem importância (não afeta a operação da interface); 1 = cosmético (não há necessidade imediata de solução); 2 = simples (problema de baixa prioridade); 3 = grave (problema de alta prioridade - deve ser reparado) e $4=$ catastrófico (muito grave, deve ser reparado de qualquer forma). ${ }^{(17)}$ Assim, se as heurísticas obtivessem classificação três ou quatro, os problemas deveriam ser corrigidos. Para selecionar os especialistas, foram adotados os seguintes critérios: ser profissional da área de tecnologias e mídias digitais, com experiência na área de jogos educativos e/ ou produção audiovisual para crianças.

Em todas as etapas de coleta de dados envolvendo seres humanos, foi solicitada a assinatura do Termo de Consentimento Livre e Esclarecido e do Termo de Assentimento pela criança. A pesquisa foi aprovada pelo Comitê de Ética em Pesquisa de um Instituto de Ensino Superior (Parecer: 1.387.962/2014) (CAAE: 63057616.7.0000.5505).

\section{Resultados}

O estudo realizado obteve como resultado a construção de um jogo de tabuleiro nomeado pelos autores como "Skuba! Uma aventura no fundo do mar". Os resultados do desenvolvimento do jogo estão descritos de acordo com as fases de realização.

$\mathrm{Na}$ fase 1 - Análise. Um estudo realizado previamente pelas autoras revelou que a criança com câncer deseja conhecer tudo o que lhe acontecerá durante o tratamento. Cada uma elenca a sua prioridade, de acordo com aquilo que vivencia. Em comum, todas as crianças acham importante que lhes contem sobre os procedimentos e os efeitos da quimioterapia. ${ }^{(1)}$

$\mathrm{Na}$ fase 2 - Conceito. Foram definidos os elementos no processo de desenvolvimento do jogo: (a) Público alvo: crianças de 8 a 12 anos, diagnosticadas com câncer e em tratamento quimioterápico; (b) Cenário do jogo: o mar, desde suas regióes mais profundas representativas do lado sombrio e desconhecido até a beleza de sua superfície. Dentro do cenário definido, os personagens do jogo foram escolhidos a partir de uma seleção de animais marinhos, que trouxeram simbologias para o público-alvo. Assim, foi possível trabalhar o conteúdo das cartas do jogo com analogias, de acordo com as características dos animais, como a capacidade de regene- 
ração da lula vampira que lembrava o crescimento do cabelo após alopecia provocada pelo tratamento, a água viva que provoca irritação na pele como o extravasamento de quimioterápicos, ou ainda, o encontro com o cavalo marinho descansando, remetendo a essa necessidade na criança; (c) dinâmica do jogo: foram incluídas cartas de sorte e de revés que representariam avanços e retrocessos dentro do jogo, além dessas acrescia-se cartas de informaçôes e cartas de negociação.

$\mathrm{Na}$ fase 3 - Design. Os elementos do jogo e seus conteúdos aprovados pelas pesquisadoras foram incorporados, resultando em um protótipo de baixa fidelidade do tabuleiro e das cartas do jogo confeccionados em papel A3.

Na fase 4 - Implementação. A partir do protótipo, uma versão-teste foi criada, impressa em papel de gramatura mais grossa, laminado e plastificado para que o jogo fosse implementado na prática com a criança. Participaram desta etapa duas crianças que jogaram partidas do jogo e revelaram a adequação das ilustraçóes das cartas, consideradas atraentes; a necessidade de modificar a posição de algumas ilustraçóes no tabuleiro; a necessidade de descrever o conteúdo de algumas cartas utilizando-se de linguagem mais acessível e clara; e a manutenção do tempo de jogo, em torno de 30 minutos. Acatadas as modificaçóes sugeridas, surgiu a versão 1 do jogo de tabuleiro.

$\mathrm{Na}$ fase 5 - Avaliação. $\mathrm{Na}$ avaliação da jogabilidade participaram cinco crianças. Da análise dos dados, emergiram duas categorias analíticas que permitiram compreender a experiência da criança ao interagir com o jogo. A primeira categoria corresponde a identificação pessoal da criança com o jogo, na qual ela se vê projetada nos elementos presentes no jogo de tabuleiro. "Eu achei interessante a lula vampira porque é igual o cabelo quando cai” (Nemo); "E a lula vampira pode se regenerar, né? Tipo, alguém corta o tentáculo dela e ela vai crescer de novo. Igual o cabelo também: se cair, cresce de novo" (Marlin).

A segunda categoria corresponde ao aprendizado da criança com o jogo. Ao interagir durante a partida, a criança tem a oportunidade de acessar, por meio das cartas, informaçóes que fazem analogias ao ambiente, aos animais marinhos, à doença e ao tratamento quimioterápico. Dessa forma, ela considera relevante o conhecimento adquirido relacionado aos personagens do jogo, bem como aos aspectos da doença e do tratamento. "Foi bom por causa das informaçóes, é um jogo de tabuleiro bom" (Gil). "Legal, porque é bem informativo, contém muitas informaçôes” (Dory).

Para validação de conteúdo participaram cinco juízes especialistas sendo três enfermeiras, uma médica oncologista pediátrica e uma pedagoga. $\mathrm{O}$ índice de validade de conteúdo geral foi de 0,95 conforme apresentado na tabela 1 .

Tabela 1. Índice de validade de conteúdo das cartas do jogo de tabuleiro pelos juízes especialistas $(n=5)$

\begin{tabular}{|c|c|}
\hline Dimensões de avaliação & IVC \\
\hline \multicolumn{2}{|l|}{ 1. Objetivos } \\
\hline 1.1 É coerente com as necessidades de informação do público-alvo. & 1 \\
\hline 1.2 É coerente, do ponto de vista educativo. & 1 \\
\hline 1.3 Pode circular no meio científico na área de oncologia pediátrica. & 1 \\
\hline $\begin{array}{l}\text { 1.4 Atende aos objetivos de profissionais e instituições que atendem/trabalham } \\
\text { com crianças com câncer. }\end{array}$ & 1 \\
\hline \multicolumn{2}{|l|}{ 2. Estrutura e apresentação } \\
\hline 2.10 jogo é apropriado para crianças com câncer em tratamento. & 1 \\
\hline 2.2 As informações das cartas estão apresentadas de maneira clara e objetiva. & 1 \\
\hline 2.3 As informações apresentadas estão cientificamente corretas. & 1 \\
\hline 2.4 Existe uma sequência lógica do conteúdo proposto. & 0,8 \\
\hline $\begin{array}{l}2.5 \text { As informações estão bem estruturadas em relação à concordância e à } \\
\text { ortografia. }\end{array}$ & 1 \\
\hline 2.6 0 estilo da redação corresponde ao nível de conhecimento do público- alvo. & 1 \\
\hline 2.7 A aparência do jogo é atrativa e adequada. & 0,8 \\
\hline 2.80 tempo de duração do jogo está adequado. & 1 \\
\hline 2.9 As ilustrações do tabuleiro são expressivas. & 0,8 \\
\hline 2.100 número de ilustrações no tabuleiro é suficiente. & 0,8 \\
\hline \multicolumn{2}{|l|}{ 3. Relevância } \\
\hline 3.1 As cartas retratam aspectos-chave que devem ser reforçados. & 1 \\
\hline $\begin{array}{l}3.20 \text { tabuleiro propõe ao jogador adquirir conhecimento sobre o tratamento } \\
\text { oncológico. }\end{array}$ & 1 \\
\hline 3.3 0 tabuleiro aborda assuntos necessários para o saber do público-alvo. & 1 \\
\hline $\begin{array}{l}3.40 \text { tabuleiro é adequado para ser usado por qualquer profissional da área de } \\
\text { oncologia pediátrica. }\end{array}$ & 1 \\
\hline Geral & 0,95 \\
\hline
\end{tabular}

$\mathrm{Na}$ avaliação dos juízes especialistas, todos os itens do instrumento tiveram um IVC acima de 0,8 na primeira rodada. Portanto, o conteúdo do jogo de tabuleiro foi considerado validado. $\mathrm{Na}$ avaliação da usabilidade participaram três profissionais experts em design de jogos educacionais. Todos atuavam na área de Tecnologia/Educação e Jogos. Os avaliadores identificaram possíveis problemas no jogo de tabuleiro e os classificaram, de acordo com a severidade do problema, conforme tabela 2 . 
Tabela 2. Avaliação do jogo de tabuleiro em relação ao grau de severidade das heurísticas de Nielsen - Primeira rodada $(n=3)$

\begin{tabular}{|c|c|c|c|c|}
\hline \multirow{2}{*}{ Heurísticas } & \multicolumn{4}{|c|}{ Grau de severidade } \\
\hline & Não identificado & Cosmético & Simples & Grave \\
\hline 1. Status do sistema & 3 & & & \\
\hline 2. Compatibilidade do sistema com o mundo real & 3 & & & \\
\hline 3. Controle do usuário e liberdade & 3 & & & \\
\hline 4. Consistência e padrões & & & 1 & 2 \\
\hline 5. Prevenção dos erros & & & & 3 \\
\hline 6. Reconhecimento ao invés de relembrança & 3 & & & \\
\hline 7. Flexibilidade e eficiência de uso & 3 & & & \\
\hline 8. Estética e design minimalista & 3 & & & \\
\hline 9. Ajuda aos usuários no reconhecimento, no diagnóstico e na correção de erros & 3 & & & \\
\hline 10.Ajuda e documentação & & 1 & 2 & \\
\hline
\end{tabular}

$\mathrm{Na}$ heurística 'consistência e padrôes', os avaliadores consideraram a coloração diferenciada de algumas casas do tabuleiro como um problema, sugerindo sua padronização. Quanto à heurística 'prevenção de erros', todos consideraram como problema de severidade grave o fato de algumas cartas resultarem em punições sequenciais em uma mesma jogada, sendo sugerida nova redação. Para a heurística 'ajuda e documentação', foram identificados problemas na redação e na ilustração dos manuais, sendo sugerida a utilização de mais recursos visuais para facilitar a compreensão do público-alvo. Todos os problemas identificados foram aceitos e encaminhados para o designer responsável para realizar as modificaçóes, sendo posteriormente submetidos a uma segunda rodada com os avaliadores que não identificaram nenhum problema de severidade grave ou catastrófica e consideraram o jogo de tabuleiro apto para ser usado pelo público-alvo.

Nesse sentido, a partir das sugestóes da população-alvo, do comitê de juízes especialistas na área temática e das alteraçôes sugeridas na avaliação das heurísticas pelos especialistas em design de jogos, foi elaborada a versão final do jogo de tabuleiro apresentada na figura 1 .

O jogo é composto de um tabuleiro, quatro pinos de cores diferentes, um dado, um guia para aplicação, um manual de regras e 114 cartas - 48, de sorte; 48, de revés; 12 , de informaçóes; e seis, de negociação. Encontra-se dividido em quatro fases que representam o pré-diagnóstico, o diagnóstico, o tratamento e o pós-tratamento. Alguns exemplos das cartas estáo representados na figura 2 e 3.

Ao vivenciar as adversidades no fundo do mar, o jogo retrata algo próximo da rotina da criança com

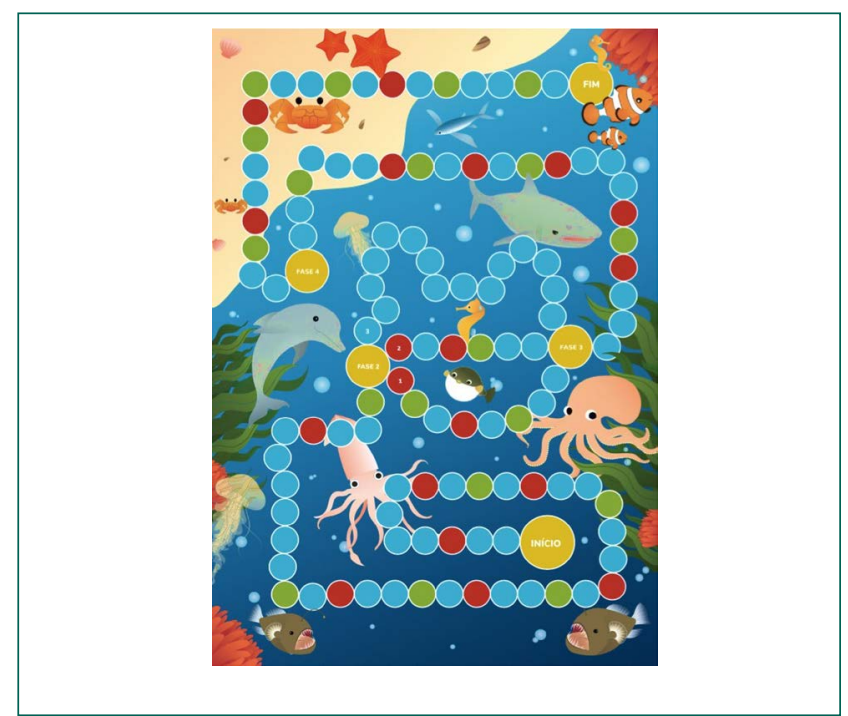

Figura 1. Jogo de tabuleiro "Skuba! Uma aventura no fundo do mar"

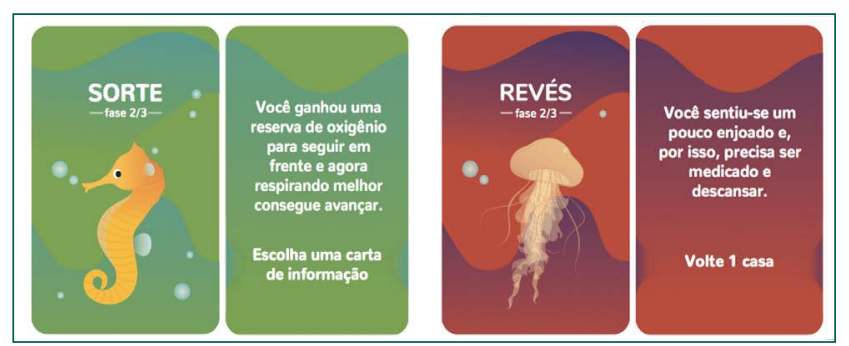

Figura 2. Exemplo de cartas de sorte e revés do jogo de tabuleiro "Skuba! Uma aventura no fundo do mar", frente e verso

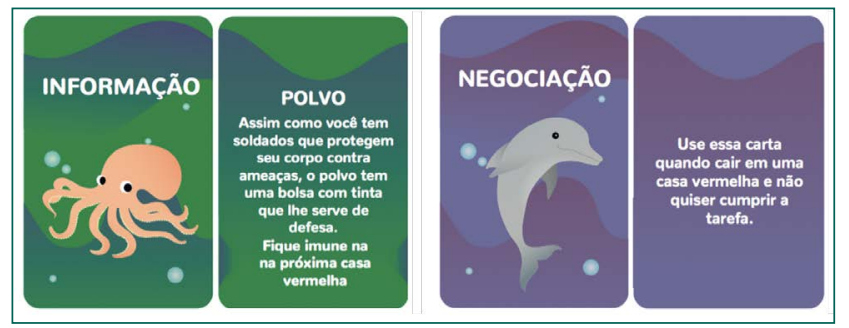

Figura 3. Exemplo de cartas de informação e negociação do jogo de tabuleiro "Skuba! Uma aventura no fundo do mar", frente e verso 
câncer, ou seja, situaçóes de ganhos e de perdas. Imersa nesse contexto, ela será desafiada a lançar mão de estratégias para superar as adversidades com que se depara.

\section{Discussão}

A criança foi ouvida nesse estudo desde a fase de análise, contribuindo com suas necessidades em relação as informaçôes a serem inseridas no jogo e trazendo importantes contribuiçóes que influenciaram na versão final do jogo de tabuleiro Skuba! Uma aventura no fundo mar.

Estudos que utilizaram o referencial metodológico adotado consideram o envolvimento como o caminho profícuo para o desenvolvimento de habilidades da criança, pois combina uma série de ações que são elementares para a aprendizagem, contribuindo para a geração de uma aprendizagem significativa. ${ }^{(18,19)}$

A confiança e o vínculo são fatores primordiais para minimizar o medo e a ansiedade. As crianças desejam ser informadas, mas a comunicação precisa acontecer por intermédio dos pais e da equipe de saúde. ${ }^{(1,5)}$ Nesse processo, estudo revela que embora o entendimento da doença do ponto de vista clínico seja, muitas vezes, mínimo, as crianças compreendem a complexidade do câncer e do tratamento e a necessidade de um esforço em equipe para atravessarem essa batalha. ${ }^{(5)}$

Dessa maneira, o referencial adotado corrobora com o processo de desenvolvimento do jogo neste estudo, cujo propósito náo foi abranger todas as informaçóes disponíveis para a criança com câncer, mas consistir num meio que pudesse intermediar a comunicação dessa com os profissionais de saúde.

Estudos realizados na área de jogos com fins lúdico-educativos tem se mostrado como uma via de comunicação considerada eficaz pelas crianças. ${ }^{(8,9,20)}$ Jogos com narrativas atraem e aproximam as crianças da equipe, despertam o interesse pelas informaçôes, além de serem uma motivação quando apresentam personagens que elas se identificam e que desejam realizar açóes similares. ${ }^{(9)}$

O processo de desenvolvimento do jogo permitiu que o resultado final fosse um produto com informaçóes simples e claras, num formato de pequenas doses nas cartas do jogo, aprovadas tanto pelas crianças como pelos especialistas. $\mathrm{O}$ conteúdo abordado foi sempre intermediado pelo lúdico, tornando a trajetória percorrida de sair do fundo do mar e atingir a superfície como uma aventura, de fato, para a criança.

Nesse contexto, os estudos tem evidenciado que os jogos cujos objetivos vão além do entretenimento constituem espaços que permitem à criança ampliar seu repertório de reaçóes, a partir da projeção do que foi vivenciado no jogo, e possibilitar uma mediação entre o lúdico e a realidade. Portanto, a utilização de recursos que tornem a comunicaçáo com a criança mais lúdica e interativa proporciona uma adaptaçáo melhor ao ambiente hospitalar e resulta em mais socialização com os profissionais que as assistem. ${ }^{(1,8,20)}$

Assim, esse estudo avança, pois destaca o uso dessa ferramenta no processo comunicacional da criança com o enfermeiro que a assiste, possibilitando uma escuta atenta e o compartilhamento de informaçóes acerca da doença e do tratamento, através de um ambiente diferenciado lúdico e dinâmico. Entretanto, faz-se necessário um preparo do enfermeiro, para lidar com os sentimentos e as dúvidas que possam surgir durante sua aplicação.

No que diz respeito às limitaçóes do estudo, destaca-se a falta de oportunidade de realizar a avaliação do jogo com um número maior de crianças que pudessem validar a sua efetividade. Assim, por meio da continuidade do uso do jogo de tabuleiro "Skuba! Uma aventura no fundo do mar" espera-se que sejam identificados aspectos a serem aperfeiçoados de maneira a torná-lo um importante instrumento de comunicação com a criança com câncer. Além disso, sugere-se, para estudos futuros, a inclusão da família no jogo, para que se avalie sua participaçáo durante as partidas com a criança.

Espera-se, portanto, que essa pesquisa instigue a aplicação na prática do jogo de tabuleiro, de maneira que haja uma translocação do conhecimento resultante e que a atuação prática do enfermeiro no cuidado dispensado à criança com câncer seja mediada por intervençóes que proporcionem efeitos benéficos a sua saúde física, cognitiva e emocional. 


\section{Conclusão}

O desenvolvimento de jogos que incluem a participação das crianças tanto no processo de concepção como de avaliação permitiu que o produto final desenvolvido fosse adequado a sua compreensão e as suas necessidades. Além disso, diferentes profissionais de áreas que vão além do contexto da saúde hospitalar podem contribuir fortemente com percepçóes diferentes ampliando discussôes e trazendo para o jogo elementos que vão além da informação, mas que o tornam mais dinâmico e divertido. A equipe de saúde precisa ver e ouvir a criança com câncer com suas necessidades e percepçóes acerca do que vivencia e o jogo de tabuleiro "Skuba! Uma aventura no fundo do mar" permite que esse processo ocorra, tornando a criança um possível participante na tomada de decisóes relativas ao processo saúde/doença.

\section{Agradecimentos}

À Coordenação de Aperfeiçoamento de Pessoal de Nível Superior (CAPES; bolsa de doutorado para Amador DD).

\section{Colaborações}

Amador DD e Mandetta MA contribuíram com a concepção do estudo, análise e interpretação dos dados, revisão do artigo, revisão crítica relevante do conteúdo intelectual e a aprovação da versão final do manuscrito.

\section{Referências}

1. Amador DD, Rodrigues LA, Mandetta MA. É melhor contar do que esconder: a informação como um direito da criança com câncer. Rev Soc Bras Enferm Ped. 2016;16(1):28-35.

2. Castro EK, Peloso F, Vital L, Armiliato MJ. Beliefs about childhood cancer: perceptions of survivors and mothers. Psicol Teor Prat. 2018;20(2):293-308.
3. Borges AA, Lima RA, Dupas G. Secrets and truths in the process of family comunication with a child with cancer. Esc Anna Nery. 2016;20(4):e20160101.

4. Sposito AM, Silva-Rodrigues FM, Sparapani VC, Pfeifer LI, de Lima RA, Nascimento LC. Coping strategies used by hospitalized children with cancer undergoing chemotherapy. J Nurs Scholarsh. 2015;47(2):14351.

5. Smith LE, Maybach AM, Feldman A, Darling A, Akard TF, Gilmer MJ. Parent and child preferences and styles of communication about cancer diagnoses and treatment. J Pediatr Oncol Nurs. 2019;36(6):390-401.

6. Sposito AM, de Montigny F, Sparapani VC, Lima RA, Silva-Rodrigues FM, Pfeifer LI, et al. Puppets as a strategy for communication with Brazilian children with cancer. Nurs Health Sci. 2016;18(1):30-7.

7. Yogman M, Garner A, Hutchinson J, Hirsh-Pasek K, Golinkoff RM; Committee on psychosocial aspects of child and family health; council on communications and media. The power of play: a pediatric role in enhancing development in young children. Pediatrics. 2018;142(3):e20182058.

8. Grigoroglou M, Papafragou A. Interactive contexts increase informativeness in children's referential communication. Dev Psychol. 2019;55(5):951-66.

9. Lu AS, Green MC, Thompson D. Using narrative game design to increase children's physical activity: exploratory thematic analysis. JMIR Serious Games. 2019;7(4):e16031.

10. Serafim AR, Silva NA, Alcântara CM, Queiroz MV. Construction of serious games for adolescents with type 1 diabetes mellitus. Acta Paul Enferm. 2019;32(4):374-81.

11. Vasileva 0, Balyasnikova N. (Re)Introducing vygotsky's thought: from historical overview to contemporary psychology. Front Psychol. 2019;10:1515. Review.

12. Moser, C. Child-Centered Game Development (CCGD): developing games with children at school. Pers Ubiquitous Comput. 2013;17(8):1647-61.

13. Quinn S, Kidd E. Symbolic play promotes non-verbal communicative exchange in infant-caregiver dyads. Br J Dev Psychol. 2019;37(1):33-50.

14. Elo S, Kyngäs $H$. The qualitative content analysis process. J Adv Nurs. 2008;62(1):107-15.

15. Pasquali L. Psychometrics. Rev Esc Enferm USP. 2009;43(Esp):992-9.

16. Alexandre NM, Coluci MZ. Validade de conteúdo nos processos de construção e adaptação de instrumentos de medidas. Cien Saude Colet. 2011;16(7):3061-8. Review.

17. Cruz AK, Neto CS. Revisitando as heurísticas de avaliação de Nielsen para análise de usabilidade em jogos de tabuleiro não virtuais. Human Factors Design. 2014;3(6):35-47.

18. Alves AG, Hostins RC. Development of imagination and creativity through the game design by children in inclusive school. Rev Bras Educ Espec. 2019;25(1):17-36.

19. Alves $A G$, Cathcart KD, Schmidt AE. Digital games as a tool for inclusive education: a case of study report. Turk Online J Educ Technology. 2015;(Spec):182-9.

20. Biddiss E, Chan-Viquez D, Cheung ST, King G. Engaging children with cerebral palsy in interactive computer play-based motor therapies: theoretical perspectives. Disabil Rehabil. 2021;43(1):133-47. 